\title{
BMJ Open Cognitive behavioural therapy in groups for medicated adults with attention deficit hyperactivity disorder: protocol for a randomised controlled trial
}

Mei-Rong Pan, ${ }^{1,2}$ Meng-Jie Zhao, ${ }^{1,2}$ Lu Liu, ${ }^{1,2}$ Hai-Mei Li, ${ }^{1,2}$ Yu-Feng Wang, ${ }^{1,2}$ Qiu-Jin Qian (1) ${ }^{1,2}$

To cite: Pan M-R, Zhao MJ, Liu L, et al. Cognitive behavioural therapy in groups for medicated adults with attention deficit hyperactivity disorder: protocol for a randomised controlled trial. BMJ Open 2020;10:e037514. doi:10.1136/ bmjopen-2020-037514

- Prepublication history for this paper is available online. To view these files, please visit the journal online (http://dx.doi. org/10.1136/bmjopen-2020037514).

Received 06 February 2020 Revised 02 August 2020 Accepted 26 August 2020
Check for updates

(c) Author(s) (or their employer(s)) 2020. Re-use permitted under CC BY-NC. No commercial re-use. See rights and permissions. Published by BMJ.

${ }^{1}$ Peking University Sixth Hospital, Institute of Mental Health,

Beijing, China

${ }^{2}$ NHC Key Laboratory of Mental Health (Peking University), National Clinical Research Center for Mental Disorders (Peking University Sixth Hospital), Beijing, China

Correspondence to

Dr Qiu-Jin Qian;

qianqiujin@bjmu.edu.cn

\section{ABSTRACT}

Introduction Cognitive behavioural therapy (CBT) is an evidence-based treatment for adults with attention deficit hyperactivity disorder (ADHD). However, it is still inconsistent whether a combination of CBT would have additive effects in medicated ADHD in adulthood. And if CBT would have additional effects, what kind and which dimension would CBT play a part? This study estimates the efficacy of CBT in stable medicated adult ADHD, using long-term outcomes and multidimensional evaluations. Methods and analysis it is a two-armed, randomised controlled trial on the superiority of the efficacy of 12 weeks of CBT on medicated adult ADHD. We compare the short-term and long-term outcomes between $\mathrm{CBT}$ combined with medication $(\mathrm{CBT}+\mathrm{M})$ group and the medication-only (M) group, including ADHD core symptoms, emotional symptoms, executive function, selfesteem, life quality and brain function using functional near-infrared spectroscopy data. Participants are outpatients of the Peking University Sixth Hospital and those recruited online, diagnosed as adult ADHD and with stable medication treatment. We estimate ADHD core symptoms and combined symptoms at baseline (T1) and week 12 (T2), week 24 (T3), week 36 (T4) and week 48 (T5).

Ethics and dissemination This trial has been approved by the Ethics and Clinical Research Committees of Peking University Sixth Hospital and will be performed under the Declaration of Helsinki with the Medical Research Involving Human Subjects Act (WMO). The results will be disseminated in a peer-reviewed journal and a conference presentation.

Trial registration number ChiCTR (ChiCTR1900021705).

\section{INTRODUCTION}

Attention deficit hyperactivity disorder (ADHD) is a childhood-onset neurodevelopmental disorder characterised by developmentally inappropriate levels of inattention, hyperactivity and impulsivity. ${ }^{1}$ About $50 \%$ of childhood ADHD have symptoms lasting into adulthood, ${ }^{2}$ affecting $2.8 \%$ of the adult

\section{Strengths and limitations of this study}

- This is the first randomised controlled trial in China to evaluate the efficacy of cognitive behaviouraltherapy (CBT) in stable medicated ADHD in adulthood, with estimations of multiple dimensions, including core symptoms, emotional status, executive function, self-esteem and social function.

- Functional near-infrared spectroscopy and executive function examinations are used to test the brain function and further exploration of the mechanism of CBT treatment.

- A 1-year follow-up is conducted to track the shortterm and long-term effects of different treatment options.

- Comorbidities and medication intake of participants need to be strictly controlled to avoid biasing test results.

- The single-centre randomised controlled study design might lead to under-represented results.

population. ${ }^{3}$ Adults with ADHD have a higher opportunity to combine with affective disorders, ${ }^{4}$ substance abuse, ${ }^{5}$ personality disorder and other psychiatric disorders. ${ }^{67}$ Meanwhile, adult ADHD encounters challenges in terms of time management, organisation, selfregulation and more occupational distress, such as lower productivity, lower income and lower job satisfaction, ${ }^{89}$ higher unemployment, ${ }^{10}$ leading to further occupational stress and economic pressure, ${ }^{11}$ poor family function and partnership. ${ }^{12}$

In clinical, the treatment of adult ADHD should follow a multimodal and multidisciplinary approach, including psychoeducation, pharmacotherapy, cognitive behavioural therapy (CBT) and coaching. ${ }^{13}$ Methylphenidate hydrochloride (MPH) and atomoxetine, mainly used in China, are almost 
equally effective for ADHD core symptoms as well as emotional symptoms, ${ }^{14} 15$ and stable medication might bring a benefit for long-term outcomes. ${ }^{16}$ However, because of side effects ${ }^{17-19}$ and residual symptoms after treatment, ${ }^{20-22}$ high treatment discontinuation and poor medication compliance are still an influencing factor for long-term outcomes. ${ }^{23} 24$

CBT has been recommended as an adjunct to medication $^{13}$ when patients are still significantly symptomatic. ${ }^{25}$ It focuses on the coherence of self-identity ${ }^{26}$ and helps patients to identify automated thinking, to clarify cognitive errors, to generate adaptive thinking with specific questioning techniques, to form better behavioural models using behavioural activation skills, to redirect emotions and actions, and ultimately to achieve symptom improvement. ${ }^{27}$ The efficacy of CBT in adult ADHD has been proven in a lot of dimensions, including selfreported ADHD core symptoms, emotional symptoms, self-esteem, social functions and time management strategies. ${ }^{28-33}$ A meta-analysis suggested that the efficacy of CBT on core symptoms could be sustained for at least 12 months. ${ }^{34}$ Whereas, up to the largest clinical trial, groups receiving psychosocial therapy had superior outcomes to active control groups at follow-up only in the Clinical Global Impression (CGI) score, but not in self-reported ADHD symptoms and emotional symptoms. ${ }^{35}$ It is demonstrated that the efficacy of CBT can be maintained up to 12 months after the end of the treatment, whereas the magnitudes of the reported effects are heterogeneous and the effect on emotional and social dimensions remains uncertain. ${ }^{29} 30343637$

Different mechanisms of action underlie pharmacotherapy and psychotherapy. ${ }^{38} \mathrm{~A}$ combination of CBT and medication theoretically might achieve a stronger clinical effect than pharmacotherapy alone. Most researches suggested that in medicated adults with ADHD, a combination of CBT would have superior efficacy on clinician-reported and self-reported ADHD symptoms, both compared with the waiting list group, ${ }^{28}{ }^{39}$ relaxation and educational support ${ }^{29}$ and clinical management $^{3037}$ groups. However, some researches showed that the CBT combined treatment showed equal success in treatment than medication alone. ${ }^{40}$ Also, inconsistent results existed when we compare the combined therapy on emotional symptoms, emotional control and social function dimensions. In some researches, self-reported depression and anxiety scores were significantly lower in the CBT group. ${ }^{28}{ }^{37}$ In Bramham et al research, clinicianreported depression and anxiety presented a significant improvement in both groups, regardless of whether the participants had received CBT. ${ }^{39}$ Philipsen et $a l^{35}$ found no significant differences in depression between groups, both after the treatment and long-term follow-up. ${ }^{41}$ And in Emilsson et al research, ${ }^{30}$ differences of emotional symptoms emerged at the 3-month follow-up but not immediately after the treatment.

In the above-mentioned studies, the inclusion criteria and medication strategies were inconsistent, which might lead to differences in clinical outcomes. Therefore, further research is needed to explore what additive effects of CBT exist in medicate-treated adults with ADHD? And what kind of clinical dimensions and neurological mechanisms may CBT and medical treatment ultimately benefit patients?

Executive dysfunctions are correlates of ADHD regardless of gender and age, ${ }^{42}$ and both stimulants ${ }^{43}$ and non-stimulants ${ }^{44}$ help to improve executive function (EF), including planning, decision making and cognitive processing capabilities. Both self-reported questionnaires and neuropsychological evaluations are necessary because different dimensions would be involved. ${ }^{45}$ In some studies, CBT helps to improve EF, mostly in areas of self-reported $\mathrm{EF}^{36}{ }^{46-48}$ whereas inconsistent in the objective evaluation index. ${ }^{47-49}$ Our team previously explored self-reported EF and some dimensions of the objective EF using Cambridge Neuropsychological Testing Automated Battery. ${ }^{47}$ Recent reviews have found inconsistent results for potential neuroimaging predictors of CBT and medical treatment outcomes in major depression, ${ }^{50} 51$ but not enough evidence for ADHD. So, it is still a question that in which way that CBT takes effect on EF, and whether there would be an objective biomarker as a potential predictor?

fNIRS is regarded as a viable alternative to functional MRI. ${ }^{5253}$ It offers the portable nature and low sensitivity to movement as well as a good resolution in both temporal and spatial domains, ${ }^{54}$ ideal for exploring brain function of the prefrontal cortex, the place where cognition and emotion interacts ${ }^{5355}$ and also predicts cognitive improvement ${ }^{5657}$ for adult ADHD. However, there are no relevant reports on the study of brain function changes before and after CBT and medical treatment using fNIRS. Based on the advantages of fNIRS, such as sensitivity, portability and time saving, results in fNIRS might be an objective test indicator for brain function before and after treatment.

Based on the previous researches of our team, the efficacy of the localised group CBT treatment module has been fully confirmed and we have accumulated a large amount of experience in the clinical study of adult ADHD. ${ }^{47}{ }^{58}$ Huang et $a l^{47}$ recruited medication naive patients and those with stable medication and followed-up for 3 months after group CBT and confirmed the efficacy of group CBT in China, including core symptoms, impulsivity and EF such as spatial planning and spatial working memory. A retrospective analysis based on the previous randomised controlled trial (RCT) and clinical statistics ${ }^{59}$ compared the efficacy of CBT with or without medication, further indicating the efficacy of group CBT for adults with ADHD in core ADHD symptoms, anxiety and depression symptoms, daily life EF and social functions. Those with stable medication presented broader improvements in self-reported EFs, but not in clinical symptoms, than CBT alone. Based on the results of previous researches, the efficacy of CBT can be confirmed, whereas RCT study is still needed to verify the further improvement of CBT in patients with stable medication. 


\section{Aim and research questions}

The primary research questions are: (1) In China, whether CBT can have additive effects on core symptoms and related symptoms in medicated adults with ADHD?, (2) What is the unique advantage of combined treatment compared with medical treatment alone? and (3) What kind of changes may happen during medical treatment and combined with CBT, especially in objective executive and brain functions? There are still other questions: (1) What specific factors contribute to the ultimate therapeutic effect during CBT or/and medication treatment, including estimated IQ, symptom severity, comorbidity, social and economic status and neuropsychological factors as biological markers? and (2) To date, a high level of heterogeneity among studies existed in the longterm outcome of CBT. ${ }^{34}$

The hypothesis of this study is that:

1. For adult ADHD with stable medication, a combination of CBT would bring an additive improvement in multiple dimensions, including core symptoms, emotional symptoms, self-esteem and functional outcomes.

2. In stable medicated adult ADHD, those combined with CBT may perform more adaptive thinking, better emotional control and quality of life over a long period of time.

3. In stable medicated adult ADHD, an improvement of brain function would be found after CBT, especially on the prefrontal lobe.

\section{METHODS AND ANALYSIS \\ Design}

To evaluate the effect of CBT on medicated ADHD adults, a two-armed, tester-blinded, superiority randomised controlled clinical trial is designed, including CBT combined with medication $(\mathrm{CBT}+\mathrm{M})$ group and the medication-only (M) group with basic clinical management based on their own need. Before the start of inclusion, the trial was registered at the Chinese Clinical Trial Registry.

The primary and secondary outcomes will be evaluated at baseline (T1), after 12 weeks of treatment (week 12, T2), week 24 (T3), week 36 (T4) and week 48 (T5). Moreover, ADHD core symptoms and two emotional symptoms will be rated weekly during the 12 weeks of treatment to monitor the changes in the $\mathrm{CBT}+\mathrm{M}$ group. The follow-up will be conducted at each time for patients in both groups from multiple dimensions (figure 1). Considering the interest of the medication-only participants, on request, we will provide them with 12 weeks of CBT at no charge after the study.

\section{Participants, recruitment and eligibility criteria}

The subjects are outpatients of the Peking University Sixth Hospital. Both patients who volunteered in outpatient clinics or those recruited through the internet will be included. All subjects will be fully informed about the

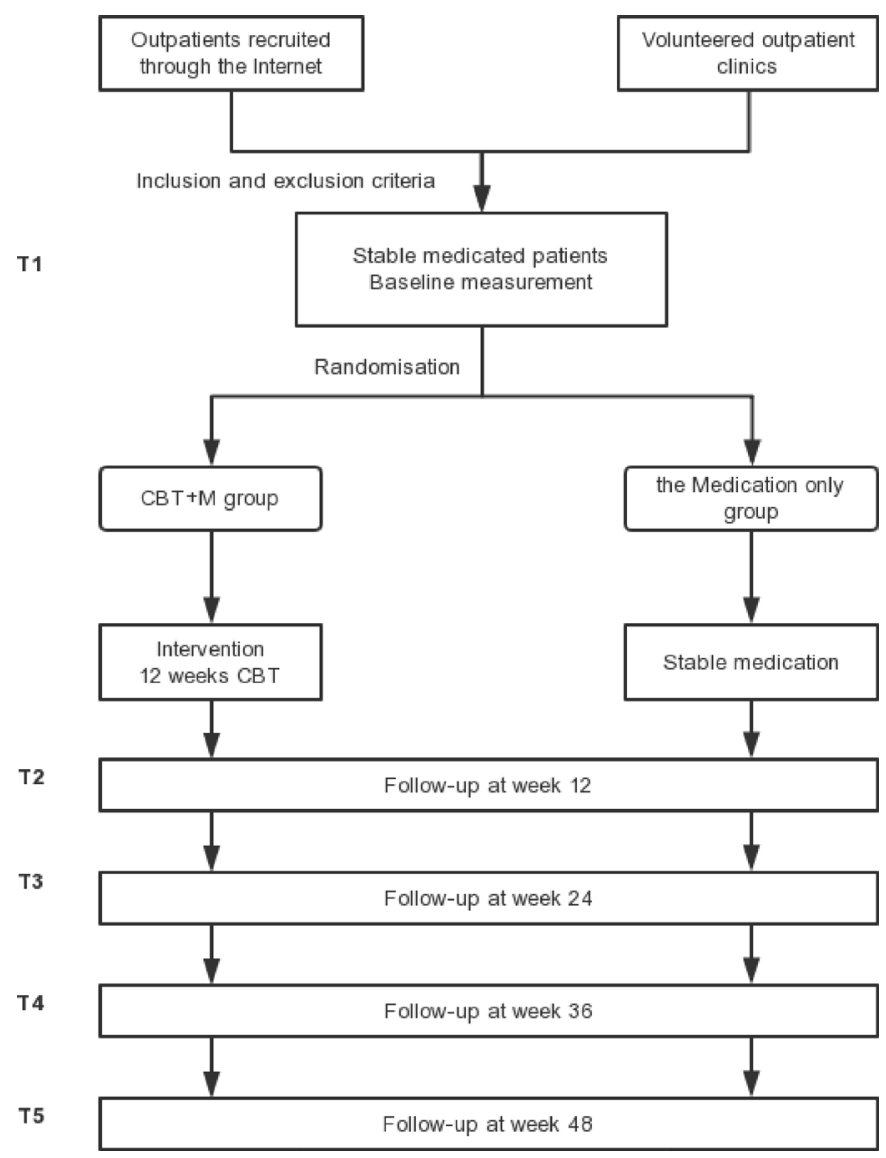

Figure 1 Trial flow. CBT, cognitive behavioural therapy; $\mathrm{CBT}+\mathrm{M}, \mathrm{CBT}$ combined with medication.

research before being asked to sign the informed consent form. The key inclusion criteria include:

1. Outpatients from Peking University Sixth Hospital, between the age of 18 and 45 years. All participants have received a diagnosis of adult ADHD through Conners' Adult ADHD Diagnostic Interview based on Diagnostic and Statistical Manual of Mental Disorders, Fourth Edition (DSM-IV), and also a Clinical Global Impression Scale (CGI-S) ${ }^{60} \geq 3$.

2. Patients with stable use of medication (drug fluctuations $<10 \%$ for at least 1 month), either with MPH controlled-release tablets (concerta) or atomoxetine hydrochloride (strattera).

The key exclusion criteria include:

1. Patients with current severe mental disorders, including psychotic disorders, current mania episode of bipolar disorder, severe depressive episodes with psychotic symptoms or high risk of suicide/self-injury, severe panic disorder, substance abuse and antisocial personality disorder.

2. Those with estimated $\mathrm{IQ}<80$.

3. Those with suicide risk.

4. Unstable physical condition (such as diabetes, angina pectoris, hypertension, active hepatitis and so on).

5. Prior or present participation in other psychological therapies. 


\section{Randomisation and blinding}

Participant randomisation will be undertaken centrally by a researcher not involved in outcome assessment or intervention delivery. The researcher uses SPSS V.22.0 software to generate random numbers and corresponding groupings, stores the sequence in a password-protected data management system and then will inform participants. All assessors and investigators (including the psychotherapists) will not be aware of which treatment order participants have been allocated to. Also, assessors at baseline and the follow-up will not be involved in the study design and CBT. Independent statisticians will analyse the research outcomes. The assessors are uniformly trained to ensure the consistency of the assessment results.

\section{Intervention}

Cognitive behavioural therapy

The 12 weeks of group CBT was based on a published and validated manual. ${ }^{28} 2961$ This manual was translated into the Chinese version and was suitable for Chinese patients. ${ }^{4762}$ The 12-weekly CBT sessions had six modules, including psychoeducation, building a helpful relationship, organisation and planning, coping with distractibility, cognitive restructuring and dealing with procrastination with all the learnt skills. As the manual was conducted individually by previous studies, we added some strategies of group CBT in practice. We selected a sizeable amount of reinforcement from the therapist and group members, making it more suitable for Chinese adult ADHD. The main structures and contents were as shown in figure 2.

There are two psychiatrists (one leader and one coleader) conducting the CBT and they have received the same systematic psychotherapy training, including CBT training and group psychotherapy training held at the Peking University Sixth Hospital and the Chinese
Psychological Society. One psychiatrist is in charge of the therapy and the other is a coleader as well as an observer. Systematic group CBT supervision is conducted at the Peking University every 2 weeks during the study to take control of therapists' competence.

The assessors are mainly postgraduate students of psychiatry, who have received unified training to use all the measurement tools. The principal investigator monitors the whole research progress and ensures all steps taken according to the original protocol. The assessment of EF and the fNIRS are conducted by one investigator respectively to minimise bias. As for diagnostic interviews and examinations, including Conners' adult ADHD diagnostic interview for DSM-IV (CAADID), Structured clinical interview for DSM-IV Axis-I disorders (SCID-I), Structured clinical interview for DSM-IV Axis-II disorders (SCID-II), Wechsler Adult Intelligence Revised in China (WAIS-RC) and so on, the investigators will be uniformly trained and consistency evaluation will be carried out after training. Kappa between assessors is between 0.76 and $0.80 \quad(p<0.01)$ to ensure the consistency of the researcher's diagnostic evaluation and meet the research needs. The Ethics Committee supervises the interests of subjects such as safety and confidentiality.

\section{Outcome assessment}

Diagnostic interview and IQ evaluations

CAADID: ${ }^{63}$ it is a structured interview based on the DSM-IV criteria and used for assessing adult ADHD diagnosis.

SCID-I and SCID-II: ${ }^{64}$ structured interview to assess disorders according to DSM-IV Axis-I (SCID-I, such as affective disorders and anxiety disorders) and Axis-II disorders (SCID-II, personality disorders) are used to assess comorbid disorders at baseline diagnosis.

WAIS-RC: ${ }^{65}$ it is divided into urban and rural versions and used to evaluate the patient's IQ. Results include

\begin{tabular}{ll}
\hline Modules & Sessions \\
\hline Psycho-education & Welcome, ice breaking and psycho-education. \\
Building a helpful relationship & Relationship of my friends and family members \\
Organisation and planning & Aims building \\
& Schedules management and multitask organisation \\
& Problem solving and major task management \\
Coping with distractibility & File organisation \\
Cognitive restructuring & Measurement of attention span and improve attentional control \\
& Management of surroundings to reduce distractibility \\
& Cognitive model introduction and it effect on ADHD \\
& Restructuring dysfunctional thinking and maladaptive beliefs \\
& An overview and practice in cognitive restructuring and emotional control \\
& Dealing with procrastination with all the learned skills \\
\hline
\end{tabular}

Figure 2 Structure for CBT treatment. ADHD, attention deficithyperactivity disorder; CBT, cognitive behavioural therapy. 
verbal IQ performance IQ and full-scale IQ (FIQ). FIQ score was used and higher scores indicate higher IQ levels.

\section{Primary outcome}

ADHD Rating Scale (ADHD-RS) ${ }^{66}$ it is a self-report 18-item scale corresponding to the DSM-IV symptoms of ADHD, including the inattention subscale and impulsiveness hyperactivity subscale. The scale is rated from 0 to 3 based on symptom frequency. Higher scores reflect more serious symptoms. The ADHD-RS was also rated by patients weekly, during the 12 weeks of CBT. The internal consistency and construct validity of ADHD-RS have been established. ${ }^{67}$

\section{Secondary outcomes}

\section{$A D H D$ core symptoms and impulsiveness}

Conners Adult ADHD Rating Scale Self-report Screening Version: ${ }^{68}$ it has 30 items and is rated from 0 to 3 scale according to the frequency of ADHD symptoms. Higher scores represent severer symptoms.

Barratt Impulsiveness Scale: ${ }^{69}$ it is a 30 -item self-report scale and widely used for self-reported impulsiveness. The items are rated 1 to 4 based on the frequency of impulsive behaviours and higher scores mean higher impulsivity. Results include three factors: attention, motor and nonplanning impulsiveness.

\section{Emotional symptoms}

Self-rating Anxiety Scale ${ }^{70}$ and Self-rating Depression Scale $^{71}$ are both 20-item self-report scales to estimate current anxiety and depressive severity. Items are rated on a scale from 1 (not at all) to 4 (most of the time) and higher total scores indicate more serious symptoms.

State-Trait Anxiety Inventory (STAI) ${ }^{72}$ and State-Trait Anger Expression Inventory-2 (STAXI-II) ${ }^{73}$ are used to estimate emotional control. STAI is a 40-item scale (20 items of anxiety state and 20 items of anxiety trait) and differentiates between the temporary condition of state anxiety and the more general and long-standing quality of trait anxiety. STAXI-II is a 57-item multidimensional measure of anger expression/control and commonly used in anger management researches.

\section{Self-esteem and social function}

Self-esteem scale: ${ }^{74}$ it is a global self-rating scale to assess the work, social life or leisure activities, and home life or family responsibilities. Items are rated on a scale from 1 to 4 and higher total scores indicate a higher level of self-esteem.

General Self-Efficacy Scale: ${ }^{75}$ it is a 10 -item self-rating scale about the assessment of self-confidence and selfefficacy in coping with difficulties. Items are rated from 1 to 4 points and higher scores indicate a higher selfefficacy. The current Chinese version of the questionnaire has good reliability and validity.

WHO Quality of Life-Brief Version (WHOQOL-BREF) ${ }^{76}$ and Sheehan Disability Scale: ${ }^{77}$ WHOQOL-BREF is a 26-item self-report scale about the degree of satisfaction in four domains, including physical health, psychological, social relationships and environment. Sheehan Disability Scale is a global self-rating scale to assess work, social life or leisure activities, and home life or family responsibilities.

\section{Automatic thoughts}

Dysfunctional Attitude Scale ${ }^{78}$ is used as a measuring tool for individuals' bad life attitudes or beliefs. Automatic Thoughts Questionnaire ${ }^{79}$ is designed to evaluate the frequency of spontaneous negative thoughts associated with depression. Coping Style Questionnaire ${ }^{80}$ is used as an assessment of a scale for measuring coping strategies.

\section{EF and brain function}

Behaviour Rating Inventory of Executive Function-Adult Version (BRIEF-A) ${ }^{81}$ it is a 75 -item self-report scale that captures views of an adult's EF or self-regulation in the patient's everyday environment, with responses scored from 1 (never) to 3 (often). Results included a composite index score, Global Executive Composite and two subindex scores: Behavioural Regulation Index and Metacognition Index, based on nine subscales. Higher scores indicate severer impairment of EF.

Stroop Colour Test, ${ }^{82}$ Trail Marking Test $\left(\right.$ TMT) ${ }^{83}$ as well as Continuous Performance Tests $(\mathrm{CPTs})^{84}$ are used to evaluate the patient's ability of selective attention, inhibition, spatial working memory, flexibility and alertness respectively.

fNIRS is used to assess the whole-brain haemodynamic changes during resting state before and after treatment.

We summarised all the evaluations and assessing time points in table 1 .

\section{Sample size}

The primary outcome measure is the reduction rate of the ADHD-RS scores. According to the results of previous research data, we defined the response to treatment as a $25 \%$ decrease in the ADHD-RS score and remission as a $40 \%$ drop in the ADHDRS-Inv score. Based on previous research ${ }^{28}$ and the formula $\mathrm{n} 1=\mathrm{n} 2=\frac{[\mathrm{z} \alpha \sqrt{2 \overline{\mathrm{p}}(1-\overline{\mathrm{p}})}+z \beta \sqrt{\mathrm{p} 1(1-\mathrm{p} 1)+\mathrm{p} 2(1-\mathrm{p} 2)}]^{2}}{(\mathrm{p} 1-\mathrm{p} 2)^{2}}$ ( $\mathrm{n}=$ number of subjects, $\mathrm{z} \alpha=1.96, \mathrm{z} \beta=1.282$ ), we assume the drop-out rate is $20 \%$ during 12 -week treatment and $30 \%$ during the follow-up sessions, so the final calculation required 46 people in each group. A total of 92 people will be included in the two groups.

\section{Data collection and management}

All clinical data, including clinical interviews and questionnaires obtained for this research, will be treated as confidential and securely stored. All written materials are entered using Epidata software. Specialised data entry staff enter the database within 1 week of the original data collection. A prompt supplement and verify is necessary if data are incomplete or unclear. Participant data will be identified by a unique ID number that will be allocated after the participant gives consent to participate 
Table 1 Evaluations applied at different time points

\begin{tabular}{|c|c|c|c|c|c|c|}
\hline Measures & $\begin{array}{l}\text { Baseline } \\
\text { (T1) }\end{array}$ & $\begin{array}{l}\text { Week } \\
2-11^{*}\end{array}$ & $\begin{array}{l}\text { Week } 12 \\
\text { (T2) }\end{array}$ & $\begin{array}{l}\text { Week } 24 \\
\text { (T3) }\end{array}$ & $\begin{array}{l}\text { Week } 36 \\
\text { (T4) }\end{array}$ & $\begin{array}{l}\text { Week } 48 \\
\text { (T5) }\end{array}$ \\
\hline Formed consent & $\bullet$ & & & & & \\
\hline Diagnostic interview and IQ evaluations & $\bullet$ & & & & & \\
\hline \multicolumn{7}{|l|}{ ADHD core symptoms } \\
\hline ADHD-RS & $\bullet$ & $\bullet$ & $\bullet$ & $\bullet$ & $\bullet$ & $\bullet$ \\
\hline CAARS & $\bullet$ & $\bullet$ & $\bullet$ & $\bullet$ & $\bullet$ & $\bullet$ \\
\hline BIS & $\bullet$ & & $\bullet$ & $\bullet$ & $\bullet$ & $\bullet$ \\
\hline \multicolumn{7}{|l|}{ Emotional symptoms } \\
\hline SAS & $\bullet$ & $\bullet$ & $\bullet$ & $\bullet$ & $\bullet$ & $\bullet$ \\
\hline SDS-1 & $\bullet$ & $\bullet$ & $\bullet$ & - & $\bullet$ & - \\
\hline STAI & $\bullet$ & & $\bullet$ & $\bullet$ & $\bullet$ & $\bullet$ \\
\hline STAIX-II & $\bullet$ & & $\bullet$ & $\bullet$ & $\bullet$ & $\bullet$ \\
\hline
\end{tabular}

Self-esteem and social function

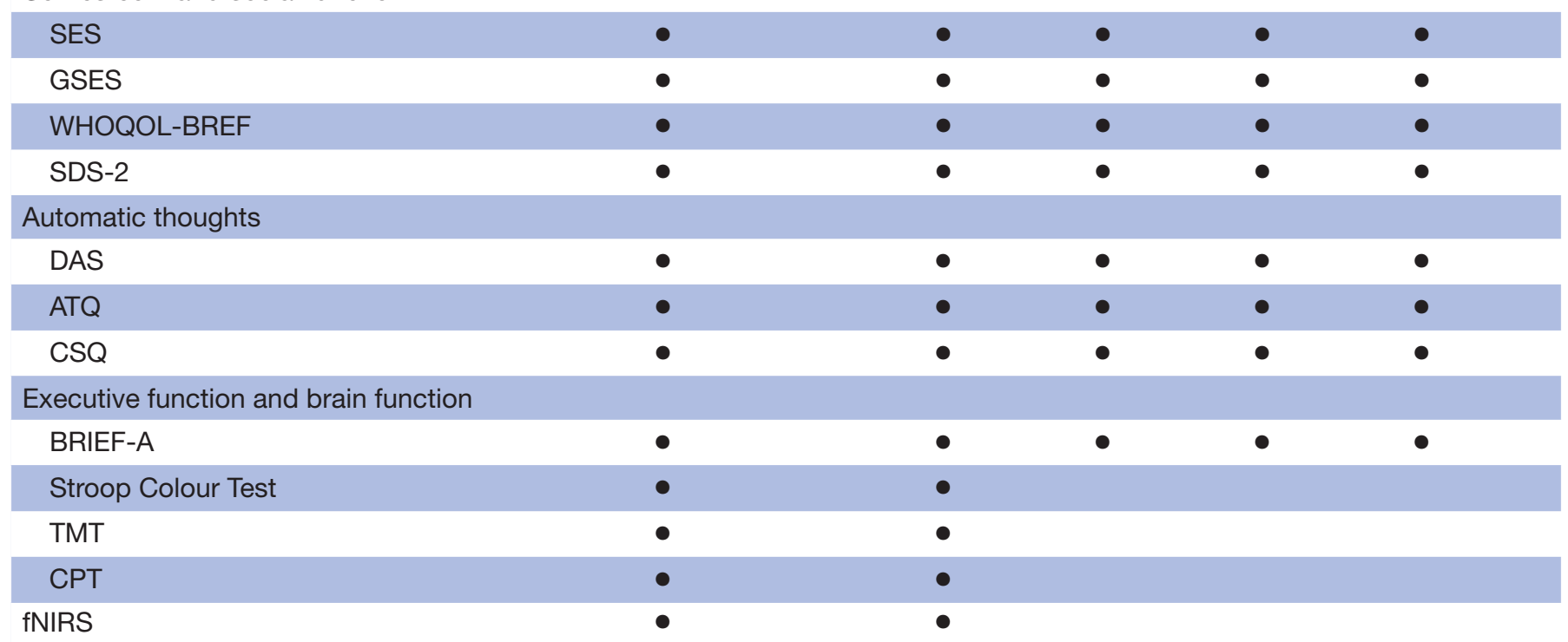

*Only for the CBT+M group.

ADHD, attention deficit hyperactivity disorder; ADHD-RS, ADHD Rating Scale; ATQ, Automatic Thoughts Questionnaire; BIS, Barratt Impulsiveness Scale; BRIEF-A, Behaviour Rating Inventory of Executive Function-Adult Version; CAARS, Conners Adult ADHD Rating Scale Self-report Screening Version; CBT, cognitive behaviroal therapy; CBT+M, CBT combined with medication; CPT, Continuous Performance Tests; CSQ, Coping Style Questionnaire; DAS, Dysfunctional Attitude Scale; fNIRS, functional near-infrared spectroscopy; GSES, General Self-Efficacy Scale; SAS, Self-rating Anxiety Scale; SDS-1, Self-rating Depression Scale; SDS-2, Sheehan Disability Scale; SES, Self-Esteem Scale; STAI, State-Trait Anxiety Inventory; STAIX-II, State-Trait Anger Expression Inventory-2; TMT, Trail Marking Test; WHOQOL-BRIEF, WHO Quality of Life-Brief Version.

in the study. The ID linking the participant's identity or personal details will be stored in a password-encrypted file located at Peking University Sixth Hospital in written and computerised formats. All electronic information will be backed up regularly to avoid loss. Only researchers affiliated with the research will have access to clinic data.

\section{Data analysis}

Two independent sample t-tests and $\chi^{2}$ tests are used to compare the baseline variables such as age, gender, IQ years of education and so on. The mixed linear model analysis (MLM) is used to compare the efficacy between groups at different estimated times. Group, time and their interaction are used as fixed effects and interindividual differences are used as random effects. We estimate each dimension at week 12 , week 24 , week 36 and week 48, including ADHD core symptoms, emotional symptoms, EF, self-esteem, automatic thinking and life quality. Moderating effects of baseline characters (such as age, gender, years of education, IQ comorbidity and so on) will also be considered. Covariate adjustment will also be conducted if significant differences at baseline between groups exist so that their effects on the results of the mixed linear model can be excluded. 
The functional connectivity analysis tool for nearinfrared spectroscopy data (FC-NIRS) package is used to preprocess the resting state data. Changes in haemoglobin concentration (oxygenated, deoxygenated and total haemoglobin) at two wavelengths (690 and $830 \mathrm{~nm})$ are calculated with modified Beer-Lambert law. To obtain low-frequency haemodynamic fluctuations, bandpass filtered is set as $0.01-0.1 \mathrm{HZ}$. Then, typical motioninduced artefacts and systematic physiological noise are removed according to temporal independent component analysis. Finally, we extract $6 \mathrm{~min}$ stable haemoglobin data from the continuous-time course of each individual. Then the mixed linear model analysis is used for further comparison.

The statistics are based on intent-to-treat analysis and the multiple imputation (imputation for five times) is conducted in the original dataset to address the missing data. The primary endpoint analysis is efficacy differences between groups with ADHD-RS scores at 12 weeks. The secondary endpoints analysis is efficacy differences with ADHD-RS scores and other measurement indicators during the follow-ups.

\section{Ethics and dissemination}

This trial has been approved by the Ethics and Clinical Research Committees of Peking University Sixth Hospital ((2018) Ethics review number (41)) and will be performed in accordance with the Declaration of Helsinki with the Medical Research Involving Human Subjects Act (WMO). Participants will have been properly informed about the study characteristics and objectives previously to study inclusion. They will also be informed about the voluntary nature of participation and the ability to leave anytime with the guarantee that they will still receive treatment in the clinic. Patients in the medication-only group will receive 12 weeks of CBT treatment with no charge once the study is finished.

\section{Patient and public involvement}

We recruit participants through online recruitment and distribution of the brochure in outpatient clinics and conduct trials with sufficient information on the study design. We will obtain written informed consent of participants before group allocation. Based on the previous researches of our team, we have solicited patients' opinions and suggestions for improvement of CBT treatment and follow-up. Following completion, a plain English version of the results will be made available to patients via their study doctor. The results will be disseminated in a peer-reviewed journal and a conference presentation.

\section{DISCUSSION}

This study has several strengths: (1) the randomised controlled design and evaluator blindness, rigorous tester training and quality control to reduce selection bias and information bias; (2) standardised diagnostic and assessment instruments including subjective self-rating scales, objective EF and brain function evaluations; (3) professional psychotherapists who have received regular training and supervision; (4) the use of the group CBT manual, which has been improved for Chinese patients and confirmed to have good therapeutic effects ${ }^{28}$; ; and (5) the short-term and long-term efficacy follow-ups.

Our study conducts multiple dimensional evaluations. Apart from ADHD core symptoms, emotional symptoms, self-esteem, automatic thinking and quality of life, we try to explore more dimensions of EF. The BRIEF-A, ${ }^{81}$ Stroop Colour Test, ${ }^{81} \mathrm{TMT}^{83}$ as well as CPTs ${ }^{85}$ could test both the ecological and practical aspects. Additionally, the NIRS measurements would be used as an objective basis for improving prefrontal function, ${ }^{8687}$ and the neuropsychological tests could help identify the potential biomarkers that reflect or predict outcomes.

Compared with previous studies in our team, we broaden the inclusion criteria in this study and included patients with affective disorders. The advantage is that the results of the study are closer to real-world clinical. However, comorbidity may also become a moderating effect, requiring an additional subgroup analysis or MLMs to examine possible moderating effects of baseline characters.

Second, we used the medication-only group as a control group, which received basic clinical management based on their own need, without specific intervention. One is to observe the natural course of the patients of medical treatment and the long-term efficacy; in contrast, the research design may lead to differences in medication adherence and clinical outcomes between groups during follow-up. Medication combined with 'non-directive supportive therapy' would be conducted in the future to reduce the bias between the groups, such as group clinical management or self-help groups.

In conclusion, this is the first time in China to study the efficacy of CBT in medication-treated adults with ADHD. Multidimensional assessment and a long-term follow-up of 48 weeks are used to figure out which dimensions patients will benefit from CBT at different periods. Moreover, an exploration of the mechanism of CBT treatment and medical treatment is needed to find the therapeutic effect factors and biomarkers and further analyse and evaluate the comprehensive intervention model of adult ADHD to provide a more individual and scientific treatment plan for adult ADHD.

Contributors All authors have contributed to the design of this trial protocol. M-RP and Q-JQ have contributed to writing the protocol and this manuscript. Q-JQ is the principal investigator of this trial and supervised the whole study. M-JZ mainly performed the assessment. LL, H-ML and Y-FW provided intellectual input to the final study protocol according to their area of expertise. All authors have contributed to the manuscript revising and approved the final manuscript.

Funding This study is funded by the National Key R\&D Program of China (2017YFC1311100), and the Capital's Funds for Health Improvement and Research (CFH 2020-2-4112; CFH 2016-2-4113).

Competing interests None declared. 
Patient and public involvement Patients and/or the public were involved in the design, or conduct, or reporting, or dissemination plans of this research. Refer to the Methods section for further details.

Patient consent for publication Not required.

Provenance and peer review Not commissioned; externally peer reviewed.

Open access This is an open access article distributed in accordance with the Creative Commons Attribution Non Commercial (CC BY-NC 4.0) license, which permits others to distribute, remix, adapt, build upon this work non-commercially, and license their derivative works on different terms, provided the original work is properly cited, appropriate credit is given, any changes made indicated, and the use is non-commercial. See: http://creativecommons.org/licenses/by-nc/4.0/.

\section{ORCID iD}

Qiu-Jin Qian http://orcid.org/0000-0001-5060-3772

\section{REFERENCES}

1 American Association Psychiatric. Diagnostic and statistical manual of mental disorders (DSM-5®). Washington: American Psychiatric Publishing, 2013

2 Lara C, Fayyad J, de Graaf R, et al. Childhood predictors of adult attention-deficit/hyperactivity disorder: results from the world Health organization world mental health survey initiative. Biol Psychiatry 2009;65:46-54.

3 Fayyad J, Sampson NA, Hwang I, et al. The descriptive epidemiology of DSM-IV adult ADHD in the world Health organization world mental health surveys. Atten Defic Hyperact Disord 2017;9:47-65.

4 Nelson JM, Liebel SW. Anxiety and depression among college students with attention-deficit/hyperactivity disorder (ADHD): Cross-informant, sex, and subtype differences. J Am Coll Health 2018;66:123-32.

5 Biederman J. Impact of comorbidity in adults with attention-deficit/ hyperactivity disorder. J Clin Psychiatry 2004;65 Suppl 3:3-7.

6 Sobanski E, Brüggemann D, Alm B, et al. Psychiatric comorbidity and functional impairment in a clinically referred sample of adults with attention-deficit/hyperactivity disorder (ADHD). Eur Arch Psychiatry Clin Neurosci 2007;257:371-7.

7 Ginsberg Y, Hirvikoski T, Lindefors N. Attention deficit hyperactivity disorder (ADHD) among longer-term prison inmates is a prevalent, persistent and disabling disorder. BMC Psychiatry 2010;10:112.

8 Mannuzza S, Klein RG. Long-Term prognosis in attention-deficit/ hyperactivity disorder. Child Adolesc Psychiatr Clin N Am 2000;9:711-26.

9 Gjervan B, Torgersen T, Nordahl HM, et al. Functional impairment and occupational outcome in adults with ADHD. J Atten Disord 2012;16:544-52.

10 Kessler RC, Adler L, Barkley R, et al. The prevalence and correlates of adult ADHD in the United States: results from the National comorbidity survey replication. Am J Psychiatry 2006;163:716-23.

11 Brod M, Schmitt E, Goodwin M, et al. ADHD burden of illness in older adults: a life course perspective. Qual Life Res 2012;21:795-9.

12 Klein RG, Mannuzza S, Olazagasti MAR, et al. Clinical and functional outcome of childhood attention-deficit/hyperactivity disorder 33 years later. Arch Gen Psychiatry 2012;69:1295-303.

13 Kooij JJS, Bijlenga D, Salerno L, et al. Updated European consensus statement on diagnosis and treatment of adult ADHD. Eur Psychiatry 2019;56:14-34.

14 Mattos P, Louzã MR, Palmini ALF, et al. A multicenter, open-label trial to evaluate the quality of life in adults with ADHD treated with long-acting methylphenidate (OROS mph): Concerta quality of life (CONQoL) study. J Atten Disord 2013;17:444-8.

15 Adler LA, Zimmerman B, Starr HL, et al. Efficacy and safety of OROS methylphenidate in adults with attention-deficit/hyperactivity disorder: a randomized, placebo-controlled, double-blind, parallel group, dose-escalation study. J Clin Psychopharmacol 2009:29:239-47.

16 Fredriksen M, Peleikis DE. Long-Term pharmacotherapy of adults with attention deficit hyperactivity disorder: a literature review and clinical study. Basic Clin Pharmacol Toxicol 2016;118:23-31.

17 Dibbets P, Evers EAT, Hurks PPM, et al. Differential brain activation patterns in adult attention-deficit hyperactivity disorder (ADHD) associated with task switching. Neuropsychology 2010;24:413-23.

18 Meijer WM, Faber A, van den Ban E, et al. Current issues around the pharmacotherapy of ADHD in children and adults. Pharm World Sci 2009;31:509-16.

19 Nair R, Moss SB. Management of attention-deficit hyperactivity disorder in adults: focus on methylphenidate hydrochloride. Neuropsychiatr Dis Treat 2009;5:421-32.
20 Safren SA, Sprich SE, Cooper-Vince C, et al. Life impairments in adults with medication-treated ADHD. J Atten Disord 2010;13:524-31.

21 Steele M, Jensen PS, Quinn DMP. Remission versus response as the goal of therapy in ADHD: a new standard for the field? Clin Ther 2006;28:1892-908.

22 Yang P-C, Lung F-W, Chiou S-S, et al. Quality of life of methylphenidate treatment-responsive adolescents with attentiondeficit/hyperactivity disorder. Kaohsiung J Med Sci 2012;28:279-84.

23 Bijlenga D, Kulcu S, van Gellecum T, et al. Persistence and adherence to psychostimulants, and psychological well-being up to 3 years after specialized treatment of adult attention-deficit/ hyperactivity disorder: a naturalistic follow-up study. J Clin Psychopharmacol 2017;37:689-96.

24 Cunill R, Castells X, Tobias A, et al. Efficacy, safety and variability in pharmacotherapy for adults with attention deficit hyperactivity disorder: a meta-analysis and meta-regression in over 9000 patients. Psychopharmacology 2016;233:187-97.

25 Dalrymple RA, McKenna Maxwell L, Russell S, et al. NICE guideline review: attention deficit hyperactivity disorder: diagnosis and management (NG87). Arch Dis Child Educ Pract Ed 2019:edpract-2019-316928.

26 Dobson KS. The science of CBT: toward a metacognitive model of change? Behav Ther 2011;2:224-7.

27 Beck AT. The past and future of cognitive therapy. J Psychother Pract Res 1997;6:276-84.

28 Safren SA, Otto MW, Sprich S, et al. Cognitive-Behavioral therapy for ADHD in medication-treated adults with continued symptoms. Behav Res Ther 2005;43:831-42.

29 Safren SA, Sprich S, Mimiaga MJ, et al. Cognitive behavioral therapy vs relaxation with educational support for medication-treated adults with ADHD and persistent symptoms: a randomized controlled trial. JAMA 2010;304:875.

30 Emilsson B, Gudjonsson G, Sigurdsson JF, et al. Cognitive behaviour therapy in medication-treated adults with ADHD and persistent symptoms: a randomized controlled trial. BMC Psychiatry 2011;11:116.

31 Weiss M, Murray C, Wasdell M, et al. A randomized controlled trial of CBT therapy for adults with ADHD with and without medication. BMC Psychiatry 2012;12:30.

32 Vidal-Estrada R, Bosch-Munso R, Nogueira-Morais M, et al. Psychological treatment of attention deficit hyperactivity disorder in adults: a systematic review. Actas Esp Psiquiatr 2012;40:147-54.

33 Young S, Emilsson B, Sigurdsson JF, et al. A randomized controlled trial reporting functional outcomes of cognitivebehavioural therapy in medication-treated adults with ADHD and comorbid psychopathology. Eur Arch Psychiatry Clin Neurosci 2017;267:267-76.

34 López-Pinar C, Martínez-Sanchís S, Carbonell-Vayá E, et al. LongTerm efficacy of psychosocial treatments for adults with attentiondeficit/hyperactivity disorder: a meta-analytic review. Front Psychol 2018;9:638.

35 Philipsen A, Jans T, Graf E, et al. Effects of group psychotherapy, individual counseling, methylphenidate, and placebo in the treatment of adult attention-deficit/hyperactivity disorder: a randomized clinical trial. JAMA Psychiatry 2015;72:1199.

36 Cherkasova MV, French LR, Syer CA, et al. Efficacy of cognitive behavioral therapy with and without medication for adults with ADHD. J Attention Dis 2017:150594641.

37 Young S, Khondoker M, Emilsson B, et al. Cognitive-Behavioural therapy in medication-treated adults with attention-deficit/ hyperactivity disorder and co-morbid psychopathology: a randomized controlled trial using multi-level analysis. Psychol Med 2015;45:2793-804.

38 Huang X, Huang P, Li D, et al. Early brain changes associated with psychotherapy in major depressive disorder revealed by restingstate fMRI: evidence for the top-down regulation theory. Int $J$ Psychophysiol 2014;94:437-44.

39 Bramham J, Young S, Bickerdike A, et al. Evaluation of group cognitive behavioral therapy for adults with ADHD. J Atten Disord 2009;12:434-41.

40 Corbisiero S, Bitto H, Newark P, et al. A Comparison of CognitiveBehavioral Therapy and Pharmacotherapy vs. Pharmacotherapy Alone in Adults With Attention-Deficit/Hyperactivity Disorder (ADHD)-A Randomized Controlled Trial. Front Psychiatry 2018;9:571.

41 Lam AP, Matthies S, Graf E, et al. Long-Term effects of multimodal treatment on adult attention-deficit/hyperactivity disorder symptoms: follow-up analysis of the COMPAS trial. JAMA Netw Open 2019;2:e194980.

42 Seidman LJ. Neuropsychological functioning in people with ADHD across the lifespan. Clin Psychol Rev 2006;26:466-85. 
43 Marraccini ME, Weyandt LL, Rossi JS, et al. Neurocognitive enhancement or impairment? A systematic meta-analysis of prescription stimulant effects on processing speed, decision-making, planning, and cognitive perseveration. Exp Clin Psychopharmacol 2016;24:269-84.

44 Gibson AP, Bettinger TL, Patel NC, et al. Atomoxetine versus stimulants for treatment of attention deficit/hyperactivity disorder Ann Pharmacother 2006;40:1134-42.

45 Low A-M, le Sommer J, Vangkilde S, et al. Delay aversion and executive functioning in adults with attention-deficit/ hyperactivity disorder: before and after stimulant treatment. Int $J$ Neuropsychopharmacol 2018;21:997-1006.

46 Fleming AP, McMahon RJ, Moran LR, et al. Pilot randomized controlled trial of dialectical behavior therapy group skills training for ADHD among college students. J Atten Disord 2015;19:260-71.

47 Huang F, Tang Y-L, Zhao M, et al. Cognitive-Behavioral therapy for adult ADHD: a randomized clinical trial in China. $J$ Atten Disord 2019;23:1035-46.

48 Mitchell JT, Mclntyre EM, English JS, et al. A pilot trial of mindfulness meditation training for ADHD in adulthood: impact on core symptoms, executive functioning, and emotion dysregulation. J Atten Disord 2017;21:1105-20.

$49 \mathrm{Gu} \mathrm{Y,} \mathrm{Xu} \mathrm{G,} \mathrm{Zhu} \mathrm{Y.} \mathrm{A} \mathrm{randomized} \mathrm{controlled} \mathrm{trial} \mathrm{of} \mathrm{Mindfulness-}$ Based cognitive therapy for college students with ADHD. J Atten Disord 2018;22:388-99.

50 Dunlop BW, Rajendra JK, Craighead WE, et al. Functional connectivity of the Subcallosal cingulate cortex and differential outcomes to treatment with cognitive-behavioral therapy or antidepressant medication for major depressive disorder. Am J Psychiatry 2017;174:533-45.

51 Chi KF, Korgaonkar M, Grieve SM. Imaging predictors of remission to anti-depressant medications in major depressive disorder. J Affect Disord 2015;186:134-44.

52 Fishburn FA, Norr ME, Medvedev AV, et al. Sensitivity of fNIRS to cognitive state and load. Front Hum Neurosci 2014;8:76.

53 Sato H, Yahata N, Funane T, et al. A NIRS-fMRI investigation of prefrontal cortex activity during a working memory task. Neuroimage 2013;83:158-73.

54 Kane RL, Parsons TD. The role of technology in clinical neuropsychology. US: Oxford University Press, 2017.

55 Aoki R, Sato H, Katura T, et al. Correlation between prefrontal cortex activity during working memory tasks and natural mood independent of personality effects: an optical topography study. Psychiatry Res 2013;212:79-87.

56 Monden Y, Dan H, Nagashima M, et al. Right prefrontal activation as a neuro-functional biomarker for monitoring acute effects of methylphenidate in ADHD children: an fNIRS study. Neuroimage Clin 2012;1:131-40

57 Ota T, lida J, Nakanishi Y, et al. Increased prefrontal hemodynamic change after atomoxetine administration in pediatric attention-deficit/ hyperactivity disorder as measured by near-infrared spectroscopy. Psychiatry Clin Neurosci 2015;69:161-70.

58 Huang F, Qian Q, Wang Y. An 24-week open-label trial for group cognitive behavioral therapy in adults with attention-deficit/ hyperactivity disorder. Chin Ment Health J 2014;12:907-12.

59 Pan M-R, Huang F, Zhao M-J, et al. A comparison of efficacy between cognitive behavioral therapy (CBT) and CBT combined with medication in adults with attention-deficit/hyperactivity disorder (ADHD). Psychiatry Res 2019;279:23-33.

60 Guy W. Clinical global impression scale, 1976.

61 Safren SA. Mastering your adult ADHD: a cognitive-behavioral treatment program: client workbook. New York Oxford: Oxford University Press, 2005

62 Huang F, Qian Q, Wang Y. Cognitive behavioral therapy for adults with attention-deficit hyperactivity disorder: study protocol for a randomized controlled trial. Trials 2015;16.

63 Conners CK, Erhardt D, Sparrow E. Conners' adult ADHD rating scales (CAARS). Toronto, Ontario, Canada: Multi-Health Systems, 1999.

64 First MB, Gibbon M. The structured clinical interview for DSM-IV axis I disorders (SCID-I) and the structured clinical interview for DSM-IV axis II disorders (SCID-II). New York: New York State Psychiatric Institute, 2004.
65 Ryan JJ, Dai X, Paolo AM. Verbal-Performance IQ discrepancies on the mainland Chinese version of the Wechsler adult intelligence scale (WAIS-RC). J Psychoeduc Assess 1995;13:365-71.

66 Dupaul GJ, Power TJ, Anastopoulos AD, et al. ADHD rating ScaleIV: checklists, norms, and clinical interpretation. J Psychoeduc Assess 2006;2:172-8.

67 Rösler M, Retz W, Thome J, et al. Psychopathological rating scales for diagnostic use in adults with attention-deficit/hyperactivity disorder (ADHD). Eur Arch Psychiatry Clin Neurosci 2006;256(Suppl 1):i3-11.

68 Conners CK, Sparrow E, Kollins SH. Guide to assessment scales in attention-deficit/hyperactivity disorder. DE: Springer Verlag, 2010.

69 Patton JH, Stanford MS, Barratt ES. Factor structure of the barratt impulsiveness scale. J Clin Psychol 1995;51:768-74.

70 Zung WW. A rating instrument for anxiety disorders. Psychosomatics 1971;12:371-9.

71 Zung WW, Richards CB, Short MJ. Self-rating depression scale in an outpatient clinic. Further validation of the SDS. Arch Gen Psychiatry 1965;6:508-15.

72 Spielberger CD, Barratt ES, Florida SU. Anxiety: current trends in theory and research. New York: Academic Press, 1972.

73 Petermann F. State- Trait- anger Expression- Inventory-2 (STAXI-2). Zeitschrift Fur Psychiatrie Psychologie Und Psychotherapie 2016;1:73-4.

74 Wang Y, Kong F, Huang L, et al. Neural correlates of biased responses: the negative method effect in the Rosenberg selfesteem scale is associated with right amygdala volume. $J$ Pers 2016;84:623-32.

75 Zhang JX, Schwarzer R. Measuring optimistic self-beliefs: a Chinese adaptation of the general self-efficacy scale. Psychologia 1995;3:174-81.

76 Skevington SM, Lotfy M, O'Connell KA. The World Health Organization's WHOQOL-BREF quality of life assessment: psychometric properties and results of the international field trial. A report from the WHOQOL group. Qual Life Res 2004;2:299-310.

77 Leu S-H, Chou J-Y, Lee P-chin, Lee PC, et al. Validity and reliability of the Chinese version of the Sheehan disability scale (SDS-C). AsiaPacific Psychiatry 2015;7:215-22.

78 Power MJ, Katz R, McGuffin P, et al. The dysfunctional attitude scale (DAS): a comparison of forms $A$ and $B$ and proposals for a new subscaled version. J Res Personal 1994;3:263-76.

79 Pan J-Y, Ye S, Ng P. Validation of the automatic thoughts questionnaire (ATQ) among mainland Chinese students in Hong Kong. J Clin Psychol 2016;72:38-48.

80 Xiao JH, XF X. Study on validity and reliability of coping style questionnaire. Chin Ment Health J 1996;04:164-8. (in Chinese).

81 Roth RM, Lance CE, Isquith PK, et al. Confirmatory factor analysis of the behavior rating inventory of executive Function-Adult version in healthy adults and application to attention-deficit/hyperactivity disorder. Arch Clin Neuropsychol 2013;28:425-34.

82 Cohn NB, Dustman RE, Bradford DC. Age-related decrements in stroop color test performance. J Clin Psychol 1984;40:1244-50.

83 Varjacic A, Mantini D, Demeyere N, et al. Neural signatures of TRAIL making test performance: evidence from lesion-mapping and neuroimaging studies. Neuropsychologia 2018;115:78-87

84 Huang-Pollock CL, Karalunas SL, Tam H, et al. Evaluating vigilance deficits in ADHD: a meta-analysis of CPT performance. J Abnorm Psychol 2012;121:360-71.

85 Ballard JC. Assessing attention: comparison of responseinhibition and traditional continuous performance tests. J Clin Exp Neuropsychol 2001;23:331-50.

86 Schecklmann M, Ehlis A-C, Plichta MM, et al. Working memory and response inhibition as one integral phenotype of adult ADHD? A behavioral and imaging correlational investigation. J Atten Disord 2013;17:470-82.

87 Yamagata B, Takei Y, Itahashi T, et al. Aberrant spatial and temporal prefrontal activation patterns in Medication-naïve adults with ADHD. Front Psychiatry 2017;274. 